\title{
Submodular optimization views on the random assignment problem
}

\author{
$\operatorname{AUTHOR}(\mathrm{S})$ :
}

Fujishige, Satoru; Sano, Yoshio; Zhan, Ping

CITATION:

Fujishige, Satoru ...[et al]. Submodular optimization views on the random assignment problem. Mathematical Programming 2019, 178(12): 485-501

\section{ISSUE DATE:}

2019-11

URL:

http://hdl.handle.net/2433/232502

\section{RIGHT:}

This is a post-peer-review, pre-copyedit version of an article published in [insert journal title]. The final authenticated version is available online at: https://doi.org/10.1007/s10107-018-1310-4.; The full-text file will be made open to the public on 21 June 2019 in accordance with publisher's 'Terms and Conditions for Self-Archiving'.; この論文は出版社版 でありません。引用の際には出版社版をご確認ご利用ください。; This is not the published version. Please cite only the published version. 


\title{
Submodular Optimization Views on the Random Assignment Problem
}

\author{
Satoru Fujishige · Yoshio Sano · Ping Zhan
}

Received: 27 June 2017; 14 June 2018 / Accepted: date

\begin{abstract}
We present a non-pricing allocation scheme of divisible goods to agents with utility functions and submodular constraints on goods. The main contribution of the present paper is that through our non-pricing allocation scheme we reveal the close relation between (1) the recent results in the allocation schemes of the random assignment problem and its extensions with ordinal or lexicographic preferences on goods and (2) the monotone algorithms of fair (egalitarian) allocations with separable utility functions and submodular constraints investigated a few decades ago. The underlying submodularity structure plays a crucial rôle, so that the probabilistic serial mechanism of Bogomolnaia and Moulin and other related mechanisms can naturally be extended to problems with submodular constraints.
\end{abstract}

Keywords Random assignment problem - Submodular optimization · Independent flows $\cdot$ Submodular flows $\cdot$ Non-pricing allocation $\cdot$ Probabilistic serial mechanism

Mathematics Subject Classification (2010) $90 \mathrm{C} 27 \cdot 52 \mathrm{~B} 40 \cdot 91 \mathrm{~B} 32 \cdot 91 \mathrm{~B} 68$

S. Fujishige's work is supported by JSPS KAKENHI Grant Numbers JP25280004 and JP26280001 and Y. Sano's work by JSPS KAKENHI Grant Numbers JP15K20885 and JP16H03118.

S. Fujishige

Research Institute for Mathematical Sciences, Kyoto University, Kyoto 606-8502, Japan.

E-mail: fujishig@kurims.kyoto-u.ac.jp

Y. Sano

Division of Information Engineering, Faculty of Engineering, Information and Systems, University of Tsukuba, Ibaraki 305-8573, Japan.

E-mail: sano@cs.tsukuba.ac.jp

P. Zhan

Department of Communication and Business, Edogawa University, Nagareyama, Chiba 270-0198, Japan.

E-mail: zhan@edogawa-u.ac.jp 


\section{Introduction}

The random assignment problem has recently been extensively investigated after the seminal paper by Bogomolnaia and Moulin [5] (see, e.g., [3,4,7, 12,18-20,23,24, $27-29,40]$ ). In the present paper we show how these results on the random assignment problem and its extensions can be viewed from submodular optimization for the fair (or egalitarian) allocation problems with convex separable utility functions and submodular constraints $[30,16,21,10,26]$ considered some decades ago.

We first consider the problem of allocating divisible goods to agents, having utility functions and submodular constraints on goods, in a fair manner without money. Related non-pricing allocation schemes have been investigated for the random assignment problem with ordinal preferences in [3, 5, 4, 7, 12,23, 24,27-29] and for allocation problems with lexicographic preferences in $[38,40]$. These results have not been extended to allocation problems with utility functions without money in general, which seems to remain open as mentioned in [40]. We propose a non-pricing allocation scheme of divisible goods to agents with utility functions and submodular constraints on goods and examine our non-pricing allocation scheme in detail.

We then show that the probabilistic serial mechanism of Bogomolnaia and Moulin [5] and its extensions [24,27,28,18-20] can be interpreted as special cases of our scheme given here for appropriately chosen utility functions. This reveals the close relation between (i) the optimal fair allocation problem and its solution algorithms in $[30,16,21,10,26]$ and (ii) the probabilistic serial mechanisms in $[5,24,27,28,18-20]$ and others, which seems to be worth further investigating. The results of the present paper furnish further insights into the behavior of the probabilistic serial mechanism and reveal the combinatorial structures that really validate it. ${ }^{1}$

The present paper is organized as follows. Section 2 provides some preliminaries on polymatroids and submodular optimization from [17] and describes the allocation problem to be considered in this paper. In Section 3 we propose a non-pricing allocation scheme as Simultaneous Monotone Algorithm and examine the solution in detail, including the case when we allow indifference on goods. Section 4 discusses implications of our non-pricing allocation scheme in the probabilistic serial mechanisms of Bogomolnaia and Moulin and its extensions and reveals the close relation between them and our non-pricing allocation scheme. Finally, Section 5 gives some concluding remarks.

\section{Preliminaries and Problem Description}

Let $N$ and $E$ be finite sets of agents and goods, respectively. For any $e \in E$ define $\chi_{e}$ to be the unit vector in $\mathbb{R}^{E}$ such that $\chi_{e}(f)=1$ if $f=e$ and $\chi_{e}(f)=0$ if $f \in E \backslash\{e\}$. For any vector $x \in \mathbb{R}^{E}$ and any set $F \subseteq E$ define $x^{F}=(x(e) \mid e \in F) \in \mathbb{R}^{F}$, the restriction of $x$ on $F$.

\footnotetext{
1 It should, however, be noted that the advantage of the random assignment problem with the probabilistic serial mechanism is that we need only agents' preferences but not their utility functions which are expensive or hard to identify in practice.
} 
2.1 Polymatroids and independent flows

We assume that we are given a polymatroid $(E, \rho)$ on $E$ with rank function $\rho: 2^{E} \rightarrow$ $\mathbb{R}_{\geq 0}$. By definition, rank function $\rho$ satisfies

(1) (normalization) $\rho(\emptyset)=0$,

(2) (monotonicity) for any $X, Y \subseteq E$ with $X \subseteq Y$ we have $\rho(X) \leq \rho(Y)$,

(3) (submodularity) for any $X, Y \subseteq E$ we have $\rho(X)+\rho(Y) \geq \rho(X \cup Y)+\rho(X \cap Y)$.

The submodular polyhedron $\mathrm{P}(\rho)$ associated with a polymatroid $(E, \rho)$ is given by

$$
\mathrm{P}(\rho)=\left\{x \in \mathbb{R}^{E} \mid \forall X \subseteq E: x(X) \leq \rho(X)\right\},
$$

where $x(X)=\sum_{e \in X} x(e)$ for any $X \subseteq E$. The intersection of $\mathrm{P}(\rho)$ and the nonnegative orthant $\mathbb{R}_{>0}^{E}$ is called the independence polytope associated with $(E, \rho)$ and is denoted by $\mathrm{P}_{(+)}(\rho)$. The base polytope $\mathrm{B}(\rho)$ associated with polymatroid $(E, \rho)$ is given by

$$
\mathrm{B}(\rho)=\{x \mid x \in \mathrm{P}(\rho), x(E)=\rho(E)\},
$$

which is always nonempty and is contained in the nonnegative orthant $\mathbb{R}_{\geq 0}^{E}$ and hence $\mathrm{B}(\rho)$ is also a face of $\mathrm{P}_{(+)}(\rho)$ (see [17] for more details about polymatroids and submodular functions).

We give some basic definitions of terms related to polymatroids that will be used in the sequel (also see [17]).

- For any set $F \subset E$ define $\rho_{F}: 2^{E \backslash F} \rightarrow \mathbb{R}_{>0}$, the contraction of $\rho$ by set $F$, by $\rho_{F}(X)=\rho(X \cup F)-\rho(F)$ for all $X \subseteq E \backslash F$, which gives another polymatroid $\left(E \backslash F, \rho_{F}\right)$ on $E \backslash F$, the contraction of $(E, \rho)$ by $F$.

- For any vector $z \in \mathrm{P}_{(+)}(\rho)$ define $\rho_{z}: 2^{E} \rightarrow \mathbb{R}_{\geq 0}$, the contraction of $\rho$ by vector $z$, by $\rho_{z}(X)=\min \{\rho(Y)-z(Y) \mid Y \supseteq X\}$ for all $X \subseteq E$, which gives another polymatroid $\left(E, \rho_{z}\right)$ on $E$, the contraction of $(E, \rho)$ by $z$. Note that for any $y \in \mathbb{R}_{>0}^{E}$, we have $y \in \mathrm{P}_{(+)}\left(\rho_{z}\right)$ if and only if $y+z \in \mathrm{P}_{(+)}(\rho)$.

- For any $\tau \in \mathbb{R}$ with $0 \leq \tau \leq \rho(E)$ define $\rho^{(\tau)}(X)=\min \{\rho(X), \tau\}$ for all $X \subseteq E$, which is called the truncation of $\rho$ by $\tau$. $\left(E, \rho^{(\tau)}\right)$ is also a polymatroid.

- Given any $x \in \mathrm{P}(\rho)$, a set $X \subseteq E$ is called $x$-tight if $x(X)=\rho(X)$. The family of $x$-tight sets is closed with respect to set union and intersection, so that there exists a unique maximal $x$-tight set, which we denote by $\operatorname{sat}(x)$. The function sat $: \mathrm{P}(\rho) \rightarrow 2^{E}$ is called the saturation function.

- Define a function dep : $\mathrm{B}(\rho) \times E \rightarrow 2^{E}$ by

$$
\operatorname{dep}(x, e)=\left\{f \in E \mid \exists \varepsilon>0: x+\varepsilon\left(\chi_{e}-\chi_{f}\right) \in \mathrm{B}(\rho)\right\}
$$

for all $x \in \mathrm{B}(\rho)$ and $e \in E$, which is called the dependence function for $(E, \rho)$.

Note that $\operatorname{dep}(x, e)$ is the unique minimal $x$-tight set containing $e \in E$.

Consider a (directed) graph $G=(V, A)$ with a vertex set $V$ and an arc set $A$, which has a set $S^{+}$of entrances and a set $S^{-}$of exits, where $S^{+}$and $S^{-}$are disjoint subsets of $V$ (see Figure 1). Each arc $a \in A$ has a capacity $c(a)>0$ and we also have a polymatroid $\mathbf{P}^{+}=\left(S^{+}, \rho^{+}\right)$on set $S^{+}$of entrances and another polymatroid $\mathbf{P}^{-}=\left(S^{-}, \rho^{-}\right)$on set $S^{-}$of exits. Denote the present network by $\mathcal{N}=(G=$ 


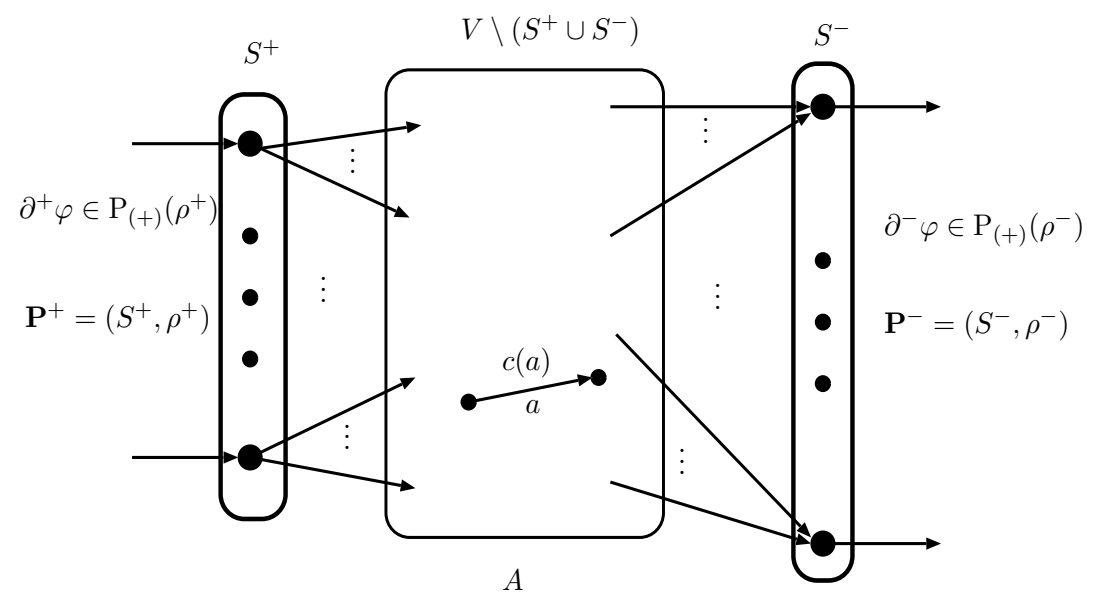

Fig. 1 An independent-flow network.

$\left.(V, A), S^{+}, S^{-}, c, \mathbf{P}^{+}=\left(S^{+}, \rho^{+}\right), \mathbf{P}^{-}=\left(S^{-}, \rho^{-}\right)\right)$. A function $\varphi: A \rightarrow \mathbb{R}$ is called an independent flow in $\mathcal{N}$ if it satisfies

$$
0 \leq \varphi(a) \leq c(a) \quad(\forall a \in A),
$$

$\partial \varphi(v)=0 \quad\left(\forall v \in V \backslash\left(S^{+} \cup S^{-}\right)\right), \quad \partial^{+} \varphi \in \mathrm{P}_{(+)}\left(\rho^{+}\right), \quad \partial^{-} \varphi \in \mathrm{P}_{(+)}\left(\rho^{-}\right)$,

where $\partial \varphi \in \mathbb{R}^{V}$ is the boundary of $\varphi$ defined by

$$
\partial \varphi(v)=\sum_{(v, u) \in A} \varphi(v, u)-\sum_{(u, v) \in A} \varphi(u, v) \quad(\forall v \in V)
$$

and $\partial^{+} \varphi \in \mathbb{R}^{S^{+}}$and $\partial^{-} \varphi \in \mathbb{R}^{S^{-}}$are defined by

$$
\partial^{+} \varphi(v)=\partial \varphi(v)\left(\forall v \in S^{+}\right), \quad \partial^{-} \varphi(v)=-\partial \varphi(v)\left(\forall v \in S^{-}\right) .
$$

(See $[15,17]$.) Also note that the model of independent flows is equivalent to that of submodular flows by Edmonds and Giles (see [17, Sect. 5]).

Concerning independent flows, we have the following proposition.

Proposition 1 Let $P \subseteq \mathbb{R}^{A}$ be the set of all independent flows in a network $\mathcal{N}=$ $\left(G=(V, A), S^{+}, S^{-}, c, \mathbf{P}^{+}=\left(S^{+}, \rho^{+}\right), \mathbf{P}^{-}=\left(S^{-}, \rho^{-}\right)\right)$. If the capacity function $c$ and the rank functions $\rho^{ \pm}$are integer-valued, then $P$ is an integral polyhedron.

Moreover, disregarding the polymatroid $\mathbf{P}^{+}=\left(S^{+}, \rho^{+}\right)$, we have the following.

Proposition 2 Let $P_{S^{+}} \subseteq \mathbb{R}^{S^{+}}$be the set of $\partial^{+} \varphi$ for all $\varphi \in \mathbb{R}^{A}$ satisfying (3) and

$$
\partial \varphi(v)=0 \quad\left(\forall v \in V \backslash\left(S^{+} \cup S^{-}\right)\right), \quad \partial^{+} \varphi \in \mathbb{R}_{\geq 0}^{S^{+}}, \quad \partial^{-} \varphi \in \mathrm{P}_{(+)}\left(\rho^{-}\right) .
$$

Then there uniquely exists a polymatroid $\left(S^{+}, \hat{\rho}\right)$ on $S^{+}$such that $P_{S^{+}}=\mathrm{P}_{(+)}(\hat{\rho})$. 
The polymatroid $\left(S^{+}, \hat{\rho}\right)$ on $S^{+}$appearing in Proposition 2 is called the polymatroid induced on $S^{+}$by $\mathbf{P}^{-}=\left(S^{-}, \rho^{-}\right)$through network $\mathcal{N}=\left(G=(V, A), S^{+}, S^{-}\right.$, $\left.c, \mathbf{P}^{-}=\left(S^{-}, \rho^{-}\right)\right)$.

\subsection{Model description}

We assume that we have a set of available good vectors $x \in \mathbb{R}_{\geq 0}^{E}$ that forms the base polytope $\mathrm{B}(\rho)$ of a polymatroid $(E, \rho)$ on $E$ with rank function $\rho: 2^{E} \rightarrow \mathbb{R}_{\geq 0}$. Also, each agent $i \in N$ has a separable convex "disutility" function $u^{i}: \mathbb{R}_{\geq 0}^{E} \rightarrow \mathbb{R}$ as

$$
u^{i}(z)=\sum_{e \in E} u_{e}^{i}(z(e))
$$

which agent $i$ wants to minimize. Here we treat convex functions to be minimized instead of concave functions to be maximized just as a matter of taste, without loss of generality. We will use the term, utility, instead of disutility in the sequel for simplicity. (We will also see in Section 4 that the minimization model fits better when we discuss its relation to the serial mechanism of the random assignment problem of Bogomolnaia and Moulin [5].) We also assume for simplicity that each $u_{e}^{i}$ is increasing and strictly convex on $[0,+\infty)$ and is differentiable on $(0,+\infty)$. Let $u_{e}^{i \prime}$ be the derivative of $u_{e}^{i}$ on $(0,+\infty)$. Note that by the assumption the set $\left\{u_{e}^{i \prime}(\alpha) \mid \alpha>0\right\}$ is an open interval, which we denote by $\left(\beta_{0}^{(i, e)}, \beta_{\infty}^{(i, e)}\right)$ where $\beta_{0}^{(i, e)} \geq 0$ and $\beta_{\infty}^{(i, e)}>0$ or $\beta_{\infty}^{(i, e)}=+\infty$. Define a function $\left(u_{e}^{i \prime}\right)^{-1}$ on $[0,+\infty)$ by

$$
\left(u_{e}^{i \prime}\right)^{-1}(\lambda)= \begin{cases}0 & \text { if } 0 \leq \lambda \leq \beta_{0}^{(i, e)} \\ \alpha & \text { if } \lambda=u_{e}^{i \prime}(\alpha) \text { for } \alpha>0 \\ +\infty & \text { if } \beta_{\infty}^{(i, e)} \leq \lambda\end{cases}
$$

for all $\lambda \in[0,+\infty)$. Note that the restriction of $\left(u_{e}^{i \prime}\right)^{-1}$ on $\left(\beta_{0}^{(i, e)}, \beta_{\infty}^{(i, e)}\right)$ is the inverse of $u_{e}^{i \prime}$ on $(0,+\infty)$. We assume that we can compute $\left(u_{e}^{i \prime}\right)^{-1}(\lambda)$ in unit time for each $\lambda \in[0,+\infty)$.

It should be emphasized that we do not allow addition of utility functions $u^{i}$ and $u^{j}$ of distinct agents $i, j \in N$ (i.e., the utilities are not transferable) though the utility functions are defined on the common space of goods where we allow addition of good vectors.

Define $\mu_{0}=\rho(E) /|N|$, which is the same (common) amount of goods for each agent to receive. We call $x=\left(x^{i}(e) \mid i \in N, e \in E\right) \in \mathbb{R}_{\geq 0}^{N \times E}$ a feasible allocation if $x$ satisfies

$$
x^{i}(E)\left(=\sum_{e \in E} x^{i}(e)\right)=\mu_{0} \quad(\forall i \in N), \quad \sum_{i \in N} x^{i} \in \mathrm{B}(\rho),
$$

where $x^{i}=\left(x^{i}(e) \mid e \in E\right) \in \mathbb{R}_{\geq 0}^{E}$ denotes a good vector that agent $i \in N$ receives. Each agent $i \in N$ wants to minimize the objective function $\sum_{e \in E} u_{e}^{i}\left(x^{i}(e)\right)$ under the overall interrelated constraints (10) for all agents. 
A feasible allocation $x=\left(x^{i}(e) \mid i \in N, e \in E\right)$ can be identified with an independent flow $\varphi: N \times E \rightarrow \mathbb{R}_{\geq 0}$ in a network $\mathcal{N}=\left(G=(V, A), S^{+}=N, S^{-}=E, d\right.$, $(E, \rho))$, where $V=N \cup E, A=N \times E$, and $d(i)=\mu_{0}(\forall i \in N)$, such that $\varphi(i, e)=x^{i}(e)$ for all $i \in N$ and $e \in E$. (See Figure 2.)

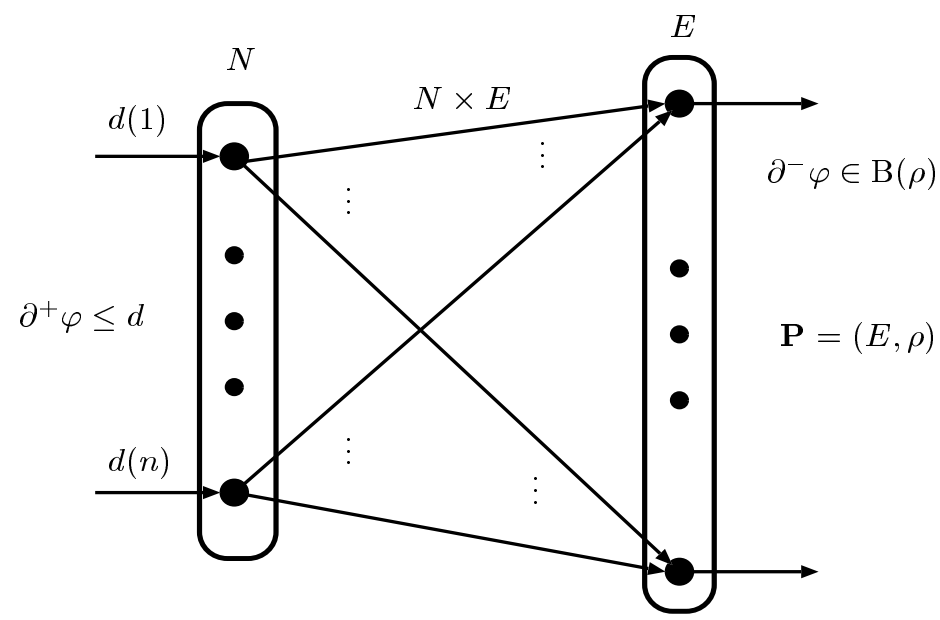

Fig. 2 A feasible allocation as an independent flow $\varphi$.

\section{Monotone Algorithms}

We propose a scheme of finding a "fair" feasible allocation of goods to agents under the constraints of (10) without money.

Suppose $N=\{1, \cdots, n\}$. We introduce some definitions and notation to be used for our algorithms given below. For each $i \in N$ define $x_{\lambda}^{i}=\left(\left(u_{e}^{i \prime}\right)^{-1}(\lambda) \mid e \in E\right)$ for all $\lambda \geq 0$. For any $\alpha \geq 0, F \subseteq E$, and $i \in N$ define $\lambda(i, F, \alpha)$ to be the value of $\lambda$ satisfying

$$
\sum_{e \in F} x_{\lambda}^{i}(e)=\alpha
$$

Note that $\lambda(i, F, \alpha)$ for each $\alpha \geq 0$ is well-defined since $x_{\lambda}^{i}(e)$ is continuous and increasing in $\lambda \in\left[0, \beta_{\infty}^{(i, e)}\right)$ and takes on all nonnegative reals. Also note that for any real $\alpha \geq 0$ equation (11) implies $0 \leq x_{\lambda}^{i}(e)<+\infty$ for all $e \in F$, and we have $u_{e}^{i \prime}\left(x_{\lambda}^{i}(e)\right)=\lambda$ (the common value $\lambda(i, F, \alpha)$ of derivatives) for all $e \in F$. 
3.1 A known result for the case of a single agent

When there is only one agent, e.g., $N=\{1\}$, there is no game-theoretic situation and the problem becomes an optimization problem:

$$
\begin{aligned}
& \mathbf{P}_{1} \text { : Minimize } \sum_{e \in E} u_{e}^{1}(x(e)) \\
& \text { subject to } x \in \mathrm{B}(\rho) \text {. }
\end{aligned}
$$

(Actually, Problem $\mathbf{P}_{1}$ was originally considered as a fair allocation problem of a single commodity to multiple agents, with $E$ being the set of agents.) It is known ([17, Sect. 9],[22]) that the following algorithm gives an optimal solution of Problem $\mathbf{P}_{1}$. Recall the notation that for any $x \in \mathbb{R}^{E}$ and $F \subseteq E$ we write $x^{F}=(x(e) \mid e \in F)$.

\section{Single Monotone Algorithm}

Input: Problem $\mathbf{P}_{1}$ with a polymatroid $(E, \rho)$ and a separable convex function $u^{1}$ : $\mathbb{R}_{>0}^{E} \rightarrow \mathbb{R}$.

Output: An optimal solution $x^{*}$ of $\mathbf{P}_{1}$.

Step 0: Put $x^{*} \leftarrow \mathbf{0} \in \mathbb{R}^{E}, k \leftarrow 0, \lambda_{0} \leftarrow 0$, and $S_{0} \leftarrow \emptyset$.

Step 1: Compute

$$
\lambda_{k+1}=\max \left\{\lambda \geq \lambda_{k} \mid x_{\lambda}^{E \backslash S_{k}} \in \mathrm{P}_{(+)}\left(\rho_{S_{k}}\right)\right\}
$$

Put $x^{*}(e) \leftarrow x_{\lambda_{k+1}}(e)$ for each $e \in E \backslash S_{k}$ and $S_{k+1} \leftarrow \operatorname{sat}\left(x^{*}\right)$.

Step 2: If $S_{k+1} \neq E$, then put $k \leftarrow k+1$ and go to Step 1 .

Otherwise return $x^{*}$.

By the procedure Single Monotone Algorithm we get a solution $x^{*}$ and a sequence of $x^{*}$-tight sets

$$
\emptyset=S_{0} \subset S_{1} \subset \cdots \subset S_{k^{*}+1}=E,
$$

where $k^{*}$ is the finally obtained index $k$. Moreover, we can easily show that

- for any distinct $e, f \in E$ such that $f \in \operatorname{dep}\left(x^{*}, e\right)$, if we have $f \in S_{k} \backslash S_{k-1}$, then $e \in E \backslash S_{k-1}$, which implies $u_{e}^{1 \prime}\left(x^{*}(e)\right) \geq u_{f}^{1 \prime}\left(x^{*}(f)\right)$.

(Recall the definition of the dependence function dep in Section 2 and note that $e \in$ $S_{k}$ implies $\operatorname{dep}\left(x^{*}, e\right) \subseteq S_{k}$.) This guarantees the optimality of the solution $x^{*}$.

Theorem $1([\mathbf{1 6 , 1 7 , 2 2 ] )}$ The procedure Single Monotone Algorithm computes an optimal solution $x^{*}$ of Problem $\mathbf{P}_{1}$. 
A special case where $u_{e}^{1}(z)=\frac{1}{w(e)} z^{2}$ with positive weights $w(e)$ for all $e \in E$ was considered in [16], which shows the monotone algorithm and a characterization of the unique optimal solution as follows. For any vector $x \in \mathbb{R}_{\geq 0}^{E}$ let $\mathbf{T}_{w}(x)$ be a linear arrangement of $x(e) / w(e)(e \in E)$ in the non-decreasing order of magnitude. That is, $\mathbf{T}_{w}(x)=\left(x\left(e_{1}\right) / w\left(e_{1}\right), \cdots, x\left(e_{m}\right) / w\left(e_{m}\right)\right)$ that satisfies $E=$ $\left\{e_{1}, \cdots, e_{m}\right\}$ and $x\left(e_{1}\right) / w\left(e_{1}\right) \leq \cdots \leq x\left(e_{m}\right) / w\left(e_{m}\right)$. We call a solution $x \in \mathrm{B}(\rho)$ a lexicographically optimal base with respect to the weight vector $w$ if $\mathbf{T}_{w}(x)$ is lexicographically maximum among linear arrangements $\mathbf{T}_{w}(y)$ for all $y \in \mathrm{B}(\rho)$. (Megiddo [30] considered a special case when $w(e)=1$ for all $e \in E$ for a polymatroid of network type, induced on a set $E$ of exits in a flow network. Also Gallo, Grigoriadis, and Tarjan [21] presented an efficient parametric algorithm for a polymatroid of network type with general positive weights as in [16].)

Theorem 2 ([16]) When $u^{1}(x)=\sum_{e \in E} \frac{1}{w(e)} x(e)^{2}$, the output $x^{*}$ of the procedure Single Monotone Algorithm is the unique lexicographically optimal base with respect to the weight vector $w$.

Informally speaking, the lexicographically optimal base $x^{*}$ is the one within the base polytope $\mathrm{B}(\rho)$ that is as proportional to $w$ as possible. The (equivalent) solution is re-discovered by Dutta and Ray [10] as a (weighted) egalitarian solution (also see $[25,26])$.

3.2 The case of multiple agents

Now let us consider the case of multiple agents, i.e., $N=\{1, \cdots, n\}$ with $n \geq 2$.

We carry out the procedure Single Monotone Algorithm simultaneously as follows. Recall the definition of $\lambda(i, F, \alpha)$ by (11) given at the beginning of Section 3 .

Simultaneous Monotone Algorithm

Input: A set $N$ of agents, a polymatroid $(E, \rho)$, and separable convex functions $u^{i}$ : $\mathbb{R}_{\geq 0}^{E} \rightarrow \mathbb{R}$ for all $i \in N$.

Output: A solution $\left(x_{*}^{i} \mid i \in N\right)$ with $x_{*}^{i} \in \mathbb{R}_{\geq 0}^{E}(i \in N)$ and $\sum_{i \in N} x_{*}^{i} \in \mathrm{B}(\rho)$.

Step 0: Put $k \leftarrow 0, \alpha_{0} \leftarrow 0, S_{0} \leftarrow \emptyset$, and $x_{*}^{i} \leftarrow \mathbf{0} \in \mathbb{R}^{E}$ for each $i \in N$.

Step 1: Putting $F_{k}=E \backslash S_{k}$, compute

$$
\alpha_{k}=\max \left\{\alpha \geq 0 \mid \sum_{i \in N}\left(x_{\lambda\left(i, F_{k}, \alpha\right)}^{i}\right)^{F_{k}} \in \mathrm{P}_{(+)}\left(\rho_{S_{k}}\right)\right\} .
$$

For each $i \in N$ and $e \in F_{k}$ put $x_{*}^{i}(e) \leftarrow x_{\lambda\left(i, F_{k}, \alpha_{k}\right)}^{i}(e)$.

Put $S_{k+1} \leftarrow \operatorname{sat}\left(\sum_{i \in N} x_{*}^{i}\right)$.

Step 2: If $S_{k+1} \neq E$, then put $k \leftarrow k+1$ and go to Step 1 .

Otherwise return $\left(x_{*}^{i} \mid i \in N\right)$. 
It should be noted that for each $i \in N$ the sequence of $\lambda\left(i, F_{k}, \alpha_{k}\right)$ for $k=$ $0,1, \ldots$ is monotone increasing, i.e.,

$$
0 \leq \lambda\left(i, F_{0}, \alpha_{0}\right)<\lambda\left(i, F_{1}, \alpha_{1}\right)<\cdots \quad(\forall i \in N)
$$

and that the output $\left(x_{*}^{i} \mid i \in N\right)$ satisfies

$$
\begin{gathered}
x_{*}^{i}(E)=\mu_{0}(\equiv \rho(E) /|N|) \quad(\forall i \in N), \\
\sum_{i \in N} x_{*}^{i} \in \mathrm{B}(\rho) .
\end{gathered}
$$

For the output $x_{*} \equiv\left(x_{*}^{i} \mid i \in N\right)$ of Simultaneous Monotone Algorithm consider the following optimization problem for each $i \in N$ :

$$
\begin{aligned}
& \mathbf{P}_{i}\left(x_{*}\right) \text { : Minimize } \sum_{e \in E} u_{e}^{i}(x(e)) \\
& \text { subject to } x \in \mathrm{B}\left(\rho_{z_{*}^{i}}\right) \text {, }
\end{aligned}
$$

where $z_{*}^{i}=\sum_{j \in N \backslash\{i\}} x_{*}^{j}$ and $\rho_{z_{*}^{i}}$ is the contraction of $\rho$ by vector $z_{*}^{i}$. It should be noted that Problem $\mathbf{P}_{i}\left(x_{*}\right)$ is to find an optimal solution for agent $i$ to choose from among $\mathrm{B}\left(\rho_{z_{*}^{i}}\right)$. In other words, if all the agents $j \in N \backslash\{i\}$ other than $i$ have received goods $x_{*}^{j}(j \in N \backslash\{i\})$ and agent $i$ can look for an optimal allocation within a set $D$ of good vectors available for agent $i$, then the set $D$ is the base polytope $\mathrm{B}\left(\rho_{z_{*}^{i}}\right)$.

Hence we have the following theorem based on the procedure Single Monotone Algorithm and Theorem 2.

Theorem 3 For the output $x_{*} \equiv\left(x_{*}^{i} \mid i \in N\right)$ of Simultaneous Monotone Algorithm, for each $i \in N$ the allocation $x_{*}^{i}$ is an optimal solution of Problem $\mathbf{P}_{i}\left(x_{*}\right)$ in (19).

For each $i \in N$ define a vector $y_{\lambda}^{i} \in \mathbb{R}^{E}$ with a parameter $\lambda \geq 0$ by

$$
y_{\lambda}^{i}(e)= \begin{cases}x_{\lambda\left(i, F_{k}, \alpha\right)}^{i}(e) & \text { if } e \in S_{k+1} \backslash S_{k}, \lambda=\lambda\left(i, F_{k}, \alpha\right) \text { for } 0 \leq \alpha \leq \alpha_{k}, \\ x_{\lambda\left(i, F_{k}, \alpha_{k}\right)}^{i}(e) & \text { if } e \in S_{k+1} \backslash S_{k}, \lambda>\lambda\left(i, F_{k}, \alpha_{k}\right)\end{cases}
$$

for all $e \in E$, where recall the definition of $\lambda(i, F, \alpha)$ by (11). Put $\lambda_{*}^{i}=\lambda\left(i, F_{k^{*}}, \alpha_{k^{*}}\right)$ where $k^{*}$ is the finally obtained index $k$. Then $y_{\lambda}^{i} \in \mathbb{R}^{E}$ with parameter $0 \leq \lambda \leq \lambda_{*}^{i}$ defines a parametric continuous increasing curve for each agent $i \in N$.

For each $i \in N$ and any $\tau$ with $0 \leq \tau \leq \mu_{0}(=\rho(E) /|N|)$ define $\lambda^{i}(\tau)$ to be the value $\lambda$ satisfying $y_{\lambda}^{i}(E)=\tau$.

From Theorem 3, we can show the following.

Theorem 4 For any $\tau$ with $0 \leq \tau \leq \rho(E),\left(y_{\lambda^{i}(\tau /|N|)}^{i} \mid i \in N\right)$ coincides with the output of Simultaneous Monotone Algorithm for the input polymatroid given by the truncation $\left(E, \rho^{(\tau)}\right)$ of $(E, \rho)$ by $\tau$, where $\rho^{(\tau)}(E)=\tau$.

(Proof) The procedure Simultaneous Monotone Algorithm generates the same intermediate allocation $\left(y_{\lambda^{i}\left(\tau^{\prime} /|N|\right)}^{i} \mid i \in N\right)$ till $\sum_{i \in N} y_{\lambda^{i}\left(\tau^{\prime} /|N|\right)}^{i}(E)$ becomes equal to $\tau$ when $\tau^{\prime}=\tau$. Hence the proof completes. 
Theorem 4 means that for each given total amount $\tau \geq 0$ of supplies the procedure Simultaneous Monotone Algorithm gives the incrementally best solution for every agent for total amount $(\tau+\Delta \tau) /|N|$ of supplies with a sufficiently small increment $\Delta \tau>0$.

\subsection{Allowing indifference on goods}

In this subsection we consider the case when there exists a collection $\mathcal{E}^{i}$ of sets of indifferent goods for each agent $i \in N$. That is, $\mathcal{E}^{i}$ is a partition $\left\{E_{1}^{i}, \cdots, E_{k_{i}}^{i}\right\}$ of $E$ for each agent $i \in N$ and the utility function $u^{i}$ is expressed as

$$
u^{i}(x)=\sum_{\ell=1}^{k_{i}} u_{\ell}^{i}\left(x\left(E_{\ell}^{i}\right)\right),
$$

where $u_{\ell}^{i}\left(\ell=1, \cdots, k_{i}\right)$ are increasing, differentiable, convex functions. Note that distinct goods $e, f \in E_{\ell}^{i}$ for $\ell \in\left\{1, \cdots, k_{i}\right\}$ are indifferent for agent $i \in N$ and the utility depends on the total amount $x\left(E_{\ell}^{i}\right)=\sum_{e \in E_{\ell}^{i}} x(e)$ but not on individual $x(e)$ for $e \in E_{\ell}^{i}$ such as $\sum_{e \in E_{\ell}^{i}} u_{\ell}^{i}(x(e))$ having the same utility function $u_{\ell}^{i}$ for all $e \in E_{\ell}^{i}$. This is modeled as an independent flow problem as follows (see [17] and Figure 3). Let $G=(V, A)$ be a graph with a vertex set $V$ and an arc set $A$ given by

$$
\begin{aligned}
V= & N \cup E \cup W, \\
& W=\left\{v_{\ell}^{i} \mid i \in N, \ell \in\left\{1, \cdots, k_{i}\right\}\right\}, \\
A= & B_{1} \cup B_{2}, \\
& B_{1}=\left\{\left(i, v_{\ell}^{i}\right) \mid i \in N, \ell \in\left\{1, \cdots, k_{i}\right\}\right\}, \\
& B_{2}=\left\{\left(v_{\ell}^{i}, e\right) \mid i \in N, \ell \in\left\{1, \cdots, k_{i}\right\}, e \in E_{\ell}^{i}\right\} .
\end{aligned}
$$

Set $N$ is the set of entrances and $E$ is the set of exits. We have a polymatroid $\mathbf{P}=$ $(E, \rho)$. Let $\varphi: A \rightarrow \mathbb{R}$ be an independent flow in the network $\mathcal{N}=\left(G=(V, A), S^{+}=N\right.$, $\left.S^{-}=E, c, d=\left(\mu_{0} \mid i \in N\right),(E, \rho)\right)$ satisfying

(a) $\forall a \in A: 0 \leq \varphi(a)<c(a)=+\infty$ (a sufficiently large number),

(b) $\forall v \in W: \partial \varphi(v)=0$,

(c) $\forall i \in N: \partial^{+} \varphi(i) \leq d(i)$ and $\partial^{-} \varphi \in \mathrm{P}_{(+)}(\rho)$.

(See Figure 3.)

Every feasible allocation $\left(x^{i} \mid i \in N\right)$ with $x^{i} \in \mathbb{R}_{\geq 0}^{E}$ is given by an independent flow $\varphi$ in $\mathcal{N}$ with $\partial^{-} \varphi \in \mathrm{B}(\rho)$ as

$$
x^{i}(e)=\varphi\left(v_{\ell}^{i}, e\right) \quad\left(i \in N, e \in E_{\ell}^{i}, \ell \in\left\{1, \cdots, k_{i}\right\}\right) .
$$

It should be noted that the value of (21) for $x=x^{i}$ is given by

$$
u^{i}\left(x^{i}\right)=\sum_{\ell=1}^{k_{i}} u_{\ell}^{i}\left(\varphi\left(i, v_{\ell}^{i}\right)\right) .
$$




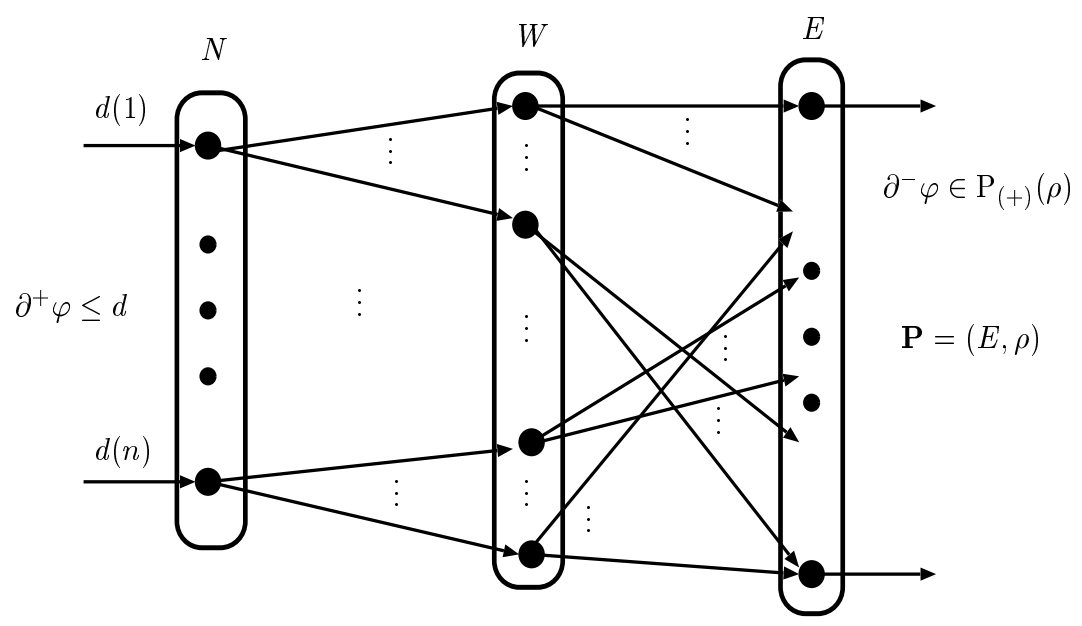

Fig. 3 An independent-flow network.

Now, for any independent flow $\varphi$ in $\mathcal{N}$ define $x_{\varphi} \in \mathbb{R}^{W}$ by

$$
x_{\varphi}(w)=\varphi\left(i, v_{\ell}^{i}\right) \quad\left(w=v_{\ell}^{i}, i \in N, \ell \in\left\{1, \cdots, k_{i}\right\}\right) .
$$

Then, from Proposition 2 we have the following.

Corollary 1 The polymatroid $(E, \rho)$ defined on the set $E$ of exits induces a polymatroid on $W$ through the subgraph $H=\left(W \cup E, B_{2}\right)$ with vertex set $W \cup E$ and arc set $B_{2}$ in (26), whose independence polytope is given by

$$
P_{W}=\left\{x_{\varphi} \mid \varphi \text { is an independent flow in } \mathcal{N}\right\} .
$$

Denote by $(W, \hat{\rho})$ the induced polymatroid in Corollary 1. It follows from (28)(30) that our problem becomes equivalent to the original allocation problem with set $N$ of agents, set $W$ of "goods", set $P_{W}=\mathrm{P}_{(+)}(\hat{\rho})$ of available "good" vectors, and utility functions (28) and without indifference. Consequently, we can employ the extended probabilistic serial mechanism shown in Section 3.2 to obtain a solution when allowing indifference. Here, every "good" is regarded as a bundle of original goods. A more general Leontief model (but with lexicographic preference orders) is considered by Schulman and Vazirani [40].

Moreover, it should be noted that the subgraph $H$ in Corollary 1 is a bipartite graph but we can deal with a general graph $\hat{H}=(\hat{V}, \hat{A})$ having the set $W$ of entrances and the set $E$ of exits with $W \cup E \subseteq \hat{V}$ and having capacities of arcs in $\hat{A}$. Similarly as in Corollary 1 a polymatroid $(W, \hat{\rho})$ is induced on $W$ from polymatroid $(E, \rho)$ on $E$. It should also be noted that for the random assignment problem, matroids of network type are considered in [7], where the underlying graph represents laminar constraints on $W$. 


\section{The Random Assignment Problem Viewed from Submodular Optimization}

In this section we examine our allocation scheme and reveal its relation to the probabilistic serial mechanism of Bogomolnaia and Moulin [5] and its extensions [27, 18, 19].

\subsection{Relation to the probabilistic serial mechanism}

Recently the probabilistic serial mechanism of Bogomolnaia and Moulin [5] has been extended from the original random assignment problem to that with polymatroidal constraints on multiple goods $[18,19]$. In this subsection we reveal that the probabilistic serial mechanism and its extensions can be interpreted as special cases of the procedure Simultaneous Monotone Algorithm given in Section 3.

We are given a polymatroid $(E, \rho)$ with integer-valued rank function $\rho$. Suppose that each agent $i \in N$ has an ordinal preference list

$$
L^{i}: e_{1}^{i} \succ_{i} \cdots \succ_{i} e_{m}^{i},
$$

where $\left\{e_{1}^{i}, \cdots, e_{m}^{i}\right\}=E$ and $e_{1}^{i}$ is agent $i$ 's best (top) good. Under the preference profile $\mathcal{L}=\left(L^{i} \mid i \in N\right)$ each agent $i$ considers $m$-tuples

$$
\left(x^{i}\left(e_{1}^{i}\right), x^{i}\left(\left\{e_{1}^{i}, e_{2}^{i}\right\}\right), \cdots, x^{i}\left(\left\{e_{1}^{i}, \cdots, e_{m}^{i}\right\}\right)\right)
$$

for all (real-valued, expected) allocations $x^{i}$ to agent $i$. Here, note that in the random assignment problem setting available good vectors are integer-valued, while by considering a lottery we have an expected real-valued allocation $\left(x^{i} \mid i \in N\right)$, which corresponds to a feasible flow in the independent-flow network $\mathcal{N}$ defined in Section 2. The (first-order) stochastic dominance relation $\succeq_{i}^{\mathrm{d}}$ over (real-valued) allocation $x^{i}$ is defined by the partial order induced by the component-wise order $\geq$ on $\mathbb{R}$ over $m$-tuples of (32). The (extended) serial mechanism to choose an efficient and envy-free solution with respect to the stochastic dominance is given as follows (paper [19] extends the Bogomolnaia-Moulin solution to the problem with submodular constraints). Define

$$
b(\mathcal{L})=\sum_{i \in N} d(i) \chi_{e_{1}^{i}}
$$

where $d(i)=\mu_{0}(=\rho(E) /|N|)$ for all $i \in N$.

\section{Extended Random Assignment}

Input: A random assignment problem $\mathbf{R A}=(N, E, \mathcal{L}, d,(E, \rho))$.

Output: A random assignment matrix $P \in \mathbb{R}_{\geq 0}^{N \times E}$.

Step 0: For each $i \in N$ put $x^{i} \leftarrow \mathbf{0} \in \mathbb{R}^{E}$ (the zero vector), and $x^{*} \leftarrow \mathbf{0} \in \mathbb{R}^{E}$. Put $S_{0} \leftarrow \emptyset, k \leftarrow 1$, and $\nu_{0} \leftarrow 0$.

Step 1: For current (updated) $\mathcal{L}=\left(L^{i} \mid i \in N\right)$, using $b(\mathcal{L})$ in (33), compute

$$
\nu_{k}=\max \left\{\nu \geq 0 \mid x^{*}+\nu b(\mathcal{L}) \in \mathrm{P}_{(+)}(\rho)\right\} .
$$


For each $i \in N$ put $x^{i} \leftarrow x^{i}+\nu_{k} d(i) \chi_{e_{1}^{i}}$.

Put $x^{*} \leftarrow x^{*}+\nu_{k} b(\mathcal{L})$ and $S_{k} \leftarrow \operatorname{sat}\left(x^{*}\right)$.

Step 2: Put $T_{k} \leftarrow S_{k} \backslash S_{k-1}$.

Update $L^{i}(i \in N)$ by removing all elements of $T_{k}$ from current $L^{i}(i \in N)$.

Step 3: If $\rho\left(S_{k}\right)<\rho(E)$, then put $k \leftarrow k+1$ and go to Step 1 .

Otherwise $\left(\rho\left(S_{k}\right)=\rho(E)\right)$ put $P(i, e) \leftarrow x^{i}(e)$ for all $i \in N$ and $e \in E$.

Return $P$.

Put $M=\max \{\rho(\{e\}) \mid e \in E\}$.

Now, reflecting the ordinal preference $L^{i}$ in (31) of every agent $i$, suppose that the utility functions $u_{e}^{i}(e \in E)$ satisfy the following condition:

$$
\beta_{0}^{\left(i, e_{\ell+1}^{i}\right)}>u_{e_{\ell}^{i}}^{i}(M) \quad(\forall \ell \in\{1, \cdots, m-1\}),
$$

where recall the definition of $\beta_{0}^{(i, e)}$ given in Section 2. Note that the right derivative of $u_{e}^{i}(z)$ at $z=0$ for $i \in N$ and $e \in E$ is equal to $\beta_{0}^{(i, e)}$.

Theorem 5 If $u_{e}^{i}(i \in N, e \in E)$ satisfy (34), then the allocation computed by Simultaneous Monotone Algorithm coincides with the solution obtained by the extended probabilistic serial mechanism Extended Random Assignment given in [19]. In particular, under assumption (34) Simultaneous Monotone Algorithm outputs the probabilistic serial solution for the original assignment problem in [5].

(Proof) Under the assumption by Simultaneous Monotone Algorithm the amounts of only the current top goods $e_{1}^{i}(i \in N)$, one for each $i$, get increased uniformly. This is exactly the procedure Extended Random Assignment of the (extended) probabilistic serial mechanism. Note that $\nu_{k}$ appearing in Step 1 of Extended Random Assignment corresponds to $\alpha_{k} / \mu_{0}$ for $\alpha_{k}$ appearing in Step 1 of Simultaneous Monotone Algorithm.

The solution obtained by Extended Random Assignment can also be interpreted as a solution of Simultaneous Monotone Algorithm for separable quadratic functions:

$$
u^{i}(x)=\sum_{e \in E} \frac{1}{w^{i}(e)} x(e)^{2} \quad(i \in N) .
$$

For each $i \in N$, corresponding to the preference order $L^{i}$ in (31), suppose that the weights $w^{i}(e)(e \in E)$ satisfy

$$
w^{i}\left(e_{1}^{i}\right) \gg w^{i}\left(e_{2}^{i}\right) \gg \cdots \gg w^{i}\left(e_{m}^{i}\right)>0 .
$$

Here, for any $a, b \in \mathbb{R} \quad a \gg b$ means that $a$ is sufficiently larger than $b$. Then Problem $\mathbf{P}_{1}$ with $u^{1}$ in (35) has an optimal solution $x^{*}$, which is as proportional to $w$ as possible. It follows from (36) that the optimal solution $x^{*}$ is obtained by lexicographically maximizing the sequence $\left(x\left(e_{1}^{1}\right), x\left(e_{2}^{1}\right), \cdots, x\left(e_{m}^{1}\right)\right)$ for $x \in \mathrm{B}(\rho)$ since we always have

$$
\mathbf{T}_{w}(x)=\left(x\left(e_{1}^{1}\right) / w^{1}\left(e_{1}^{1}\right), x\left(e_{2}^{1}\right) / w^{1}\left(e_{2}^{1}\right), \cdots, x\left(e_{m}^{1}\right) / w^{1}\left(e_{m}^{1}\right)\right)
$$


for all $x \in \mathrm{B}(\rho)$. Hence $x^{*}$ is the optimal solution computed by what is called the greedy algorithm of Edmonds [11] as

$$
x^{*}\left(e_{\ell}^{1}\right)=\rho\left(\left\{e_{1}^{1}, \cdots, e_{\ell}^{1}\right\}\right)-\rho\left(\left\{e_{1}^{1}, \cdots, e_{\ell-1}^{1}\right\}\right) \quad(\ell=1, \cdots, m) .
$$

It should be noted that from (38) sets $\left\{e_{1}^{1}, \cdots, e_{\ell}^{1}\right\}(\ell=1, \cdots, m)$ are $x^{*}$-tight, so that we have

$$
x^{*}\left(\left\{e_{1}^{1}, \cdots, e_{\ell}^{1}\right\}\right)=\rho\left(\left\{e_{1}^{1}, \cdots, e_{\ell}^{1}\right\}\right) \geq y\left(\left\{e_{1}^{1}, \cdots, e_{\ell}^{1}\right\}\right) \quad(\ell=1, \cdots, m)
$$

for all $y \in \mathrm{B}(\rho)$. In other words, $x^{*}$ is the maximum vector in $\mathrm{B}(\rho)$ with respect to the (first-order) stochastic dominance relation associated with the preference order $L^{1}$ in (31).

Consequently, we may call the procedure Simultaneous Monotone Algorithm for (35) and (36) a simultaneous greedy algorithm in the sense of Edmonds. We can restate Theorem 3 as follows.

Corollary 2 Under the assumptions (35) and (36), for the output $x_{*} \equiv\left(x_{*}^{i} \mid i \in N\right)$ of Simultaneous Monotone Algorithm, for each $i \in N$ the allocation $x_{*}^{i}$ is the one obtained by the greedy algorithm of Edmonds on base polytope $\mathrm{B}\left(\rho_{z_{*}^{i}}\right)$.

\subsection{Quasi-order preferences}

Katta and Sethuraman [27] considered the random assignment problem on the full preference domain of quasi-orders, where we have a partition $\mathcal{E}^{i}=\left\{E_{1}^{i}, \cdots, E_{k_{i}}^{i}\right\}$ of $E$ (into the indifference class of sets) for each agent $i \in N$ and the preference order list $L^{i}$ on $\mathcal{E}^{i}$ as

$$
L^{i}: E_{1}^{i} \succ_{i} \cdots \succ_{i} E_{k_{i}}^{i} .
$$

A special case of dichotomous preferences was considered earlier by Bogomolnaia and Moulin [6]. Allowing indifference for goods, the problem with utility functions can be reduced to a problem without indifference as shown in Section 3.3. For the induced problem we can further apply the result of Section 4.1 to deal with the preference orders (40).

We can easily see that the output of Simultaneous Monotone Algorithm with $u^{i}(i \in N)$ modified in Section 3.3 coincides with the solution obtained by the probabilistic serial mechanism of Katta and Sethuraman [27] (extended to the problem with polymatroidal constraints).

This explains why the flow algorithms in $[30,16,21]$ appear in the mechanism of Katta and Sethuraman [27] (also [6]) as a subroutine.

\subsection{Non-uniform shares}

We have assumed that the total amount $\rho(E)$ of goods are uniformly distributed to all agents, each receiving $\mu_{0}=\rho(E) /|N|$ of $\rho(E)$.

Now suppose that we are given $\mu_{0}^{i}>0(i \in N)$ satisfying $\sum_{i \in N} \mu_{0}^{i}=\rho(E)$ and that each agent $i \in N$ receives the total amount $\mu_{0}^{i}$ of goods. Then we adapt 
the procedure Simultaneous Monotone Algorithm in such a way that the output satisfies the requirement as follows.

We modify the definition (11) of $\lambda(i, F, \alpha)$ alone. Other definitions are the same as in Section 3.2. For any $i \in N, F \subseteq E$, and $\alpha$ with $0 \leq \alpha \leq 1$ define $\lambda(i, F, \alpha)$ to be the value of $\lambda$ satisfying

$$
\sum_{e \in F} x_{\lambda}^{i}(e)=\mu_{0}^{i} \alpha .
$$

Then Theorems 3 and 4 hold mutatis mutandis.

\section{Concluding Remarks}

We have presented a solution for the non-pricing allocation of divisible goods to agents with utility functions and submodular constraints and showed the adaptability of the probabilistic serial mechanism of Bogomolnaia and Moulin [5] to the nonpricing allocation of divisible goods to agents with utility functions. Our results reveal how the probabilistic serial mechanism of Bogomolnaia and Moulin [5] and its extensions [27, 18-20] are related to the optimal fair allocation algorithms of Megiddo [30], Fujishige [16], Gallo, Grigoriadis, and Tarjan [21], and Groenevelt [22] and to the greedy algorithm of Edmonds [11]. This also shows that it is natural to consider extensions of the original assignment problem to those with sets of available good vectors expressed by submodular functions as in [18-20].

Apart from the submodular optimization views on the probabilistic serial mechanisms, our simultaneous monotone algorithm can be employed as a non-pricing allocation scheme when we are given such a fair allocation problem with non-transferable separable convex utility functions and submodular constraints to be solved. Then we have to examine the computational complexity issue of our algorithm in more detail. Also a game-theoretical issue remains to be investigated when every agent does not necessarily reveal her utility function truthfully.

Although the models and goals are different, it is also worth mentioning that there exist a lot of related researches on fair allocation problems such as those in $[1,2,8,9,13,14,31-34]$ among others. The submodular optimization views given in the present paper are expectedly useful for further investigation of the models and allocation schemes treated there.

Throughout the present paper the separability of utility functions plays a crucial rôle. How to deal with non-separable utility functions is also left open. It seems that $\mathrm{M}^{\natural}$-convex functions ([35]) are good candidates for utility functions without separability in order to extend our allocation scheme appropriately, since separable convex functions defined on independence polytopes (polymatroid polytopes) are $\mathrm{M}^{\natural}$-convex (see [35-37,39] for more details).

\section{Acknowledgments}

We are very grateful to Britta Peis for communications and useful discussions about the subject of this paper, which motivated our present work and improved the presentation. We also appreciate useful comments of an anonymous referee. 


\section{References}

1. O. Bochet, R. İlkılıç, and H. Moulin: Egalitarianism under earmark constraints. Journal of Economic Theory 148 (2013) 535-562.

2. O. Bochet, R. İlkılıç, H. Moulin, and J. Sethuraman: Balancing supply and demand under bilateral constraints. Theoretical Economics 7 (2012) 395-423.

3. A. Bogomolnaia: Random assignment: redefining the serial rule. Journal of Economic Theory 158 (2015) 308-318.

4. A. Bogomolnaia and E. J. Heo: Probabilistic assignment of objects: characterizing the serial rule. Journal of Economic Theory 147 (2012) 2072-2082.

5. A. Bogomolnaia and H. Moulin: A new solution to the random assignment problem. Journal of Economic Theory 100 (2001) 295-328.

6. A. Bogomolnaia and H. Moulin: Random matching under dichotomous preferences. Econometrica 72 (2004) 257-279.

7. E. Budish, Y.-K. Che, F. Kojima, and P. Milgrom: Designing random allocation mechanisms: theory and applications. American Economic Review 103 (2013) 585-623.

8. S. Chandramouli and J. Sethuraman: Groupstrategyproofness of the egalitarian mechanism for constrained rationing problems. Mathematical Social Sciences 90 (2017) 111-118.

9. Y.-K. Che, J. Kim, and K. Mierendorff: Generalized reduced-form auctions: a network-flow approach. Econometrica 81 (2013) 2487-2520.

10. B. Dutta and D. Ray: A concept of egalitarianism under participation constraints. Econometrica 57 (1989) 615-635.

11. J. Edmonds: Submodular functions, matroids, and certain polyhedra. Proceedings of the Calgary International Conference on Combinatorial Structures and Their Applications (R. Guy, H. Hanani, N. Sauer and J. Schönheim, eds., Gordon and Breach, New York, 1970), pp. 69-87.

12. Ö. Ekici and O. Kesten: An equilibrium analysis of the probabilistic serial mechanism. International Journal of Game theory 45 (2016) 655-674.

13. K. Flores-Szwagrzak: The replacement principle in networked economies with single-peaked preferences. Social Choice and Welfare 47 (2016) 763-789.

14. K. Flores-Szwagrzak: Efficient, fair, and strategy-proof (re)allocation under network constraints. Social Choice and Welfare 48 (2017) 109-131.

15. S. Fujishige: Algorithms for solving the independent-flow problems. Journal of the Operations Research Society of Japan 21 (1978) 189-204.

16. S. Fujishige: Lexicographically optimal base of a polymatroid with respect to a weight vector. Mathematics of Operations Research 2 (1980) 186-196.

17. S. Fujishige: Submodular Functions and Optimization Second Edition (Elsevier, 2005).

18. S. Fujishige, Y. Sano, and P. Zhan: A solution to the random assignment problem with a matroidal family of goods. RIMS Preprint RIMS-1852, Kyoto University, May 2016.

19. S. Fujishige, Y. Sano, and P. Zhan: An extended probabilistic serial mechanism to the random assignment problem with multi-unit demands and polymatroidal supplies. RIMS Preprint RIMS-1866, Kyoto University, November 2016

20. S. Fujishige, Y. Sano, and P. Zhan: The random assignment problem with submodular constraints on goods. ACM Transactions on Economics and Computation 6, 1, Article 3 (March 2018), 28 pages.

21. G. Gallo, M. D. Grigoriadis, and R. E. Tarjan: A fast parametric maximum flow algorithm and applications. SIAM Journal on Computing 18 (1989) 30-55.

22. H. Groenevelt: Two algorithms for maximizing a separable concave function over a polymatroid feasible region. European Journal of Operational Research 54 (1991) 227-236.

23. T. Hashimoto, D. Hirata, O. Kesten, M. Kurino, and M. U. Ünver: Two axiomatic approaches to the probabilistic serial mechanism. Theoretical Economics 9 (2014) 253-277.

24. E. J. Heo: Probabilistic assignment problem with multi-unit demands: a generalization of the serial rule and its characterization. Journal of Mathematical Economics 54 (2014) 40-47.

25. T. Hokari: Monotone-path Dutta-Ray solution on convex games. Social Choice and Welfare 19 (2002) $825-844$.

26. T. Hokari and A. van Gellekom: Population monotonicity and consistency in convex games: Some logical relations. International Journal of Game Theory 31 (2002) 593-607.

27. A.-K. Katta and J. Sethuraman: A solution to the random assignment problem on the full preference domain. Journal of Economic Theory 131 (2006) 231-250. 
28. F. Kojima: Random assignment of multiple indivisible objects. Mathematical Social Sciences $\mathbf{5 7}$ (2009) 134-142.

29. F. Kojima and M. Manea: Incentives in the probabilistic serial mechanism. Journal of Economic Theory 145 (2010) 106-123.

30. N. Megiddo: Optimal flows in networks with multiple sources and sinks. Mathematical Programming 7 (1974) 97-107.

31. H. Moulin: Entropy, desegregation, and proportional rationing. Journal of Economic Theory $\mathbf{1 6 2}$ (2016) $1-20$.

32. H. Moulin: Consistent bilateral assignment. Mathematical Social Sciences 90 (2017) 43-55.

33. H. Moulin: One-dimensional mechanism design. Theoretical Economics 12 (2017) 587-619.

34. H. Moulin and Sethuraman: The bipartite rationing problem. Operations Research 61 (2013) 1087 1100 .

35. K. Murota: Discrete Convex Analysis (SIAM Monographs on Discrete Mathematics and Applications 10, SIAM, 2003).

36. K. Murota: Discrete convex analysis: a tool for economics and game theory. Journal of Mechanism and Institution Design 1 (2016) 151-273.

37. K. Murota and A. Shioura: M-convex function on generalized polymatroid. Mathematics of Operations Research 24 (1999) 95-105.

38. D. Saban and J. Sethuraman: A note on object allocation under lexicographic preferences. Journal of Mathematical Economics 50 (2014) 283-289.

39. A. Shioura and A. Tamura: Gross substitutes condition and discrete concavity for multi-unit valuations: a survey. Journal of the Operations Research Society of Japan 58 (2015) 61-103.

40. L. J. Schulman and V. V. Vazirani: Allocation of divisible goods under lexicographic preferences. Leibniz International Proceedings in Informatics (35th IARCS Annual Conf. Foundation of Software Technology and Theoretical Computer Science (FSTTCS 2015)) (Eds., P. Harsha and G. Ramalingam), pp. 543-559. 